\title{
Adaptation of Inductive Energy Store on Diesel Railway Locomotive
}

\author{
M.E. Vilberger \\ Novosibirsk State Technical University \\ Novosibirsk, Russian Federation \\ e-mail: ksmetk@mail.ru \\ B.V. Malozyomov \\ Novosibirsk State Technical University \\ Novosibirsk, Russian Federation \\ e-mail:mbv5@mail.ru
}

\author{
V.V. Zhulovyan \\ Novosibirsk State Technical University \\ Novosibirsk, Russian Federation \\ e-mail: ksm3@mail.ru \\ I.S. Demidenko \\ Novosibirsk State Technical University \\ Novosibirsk, Russian Federation \\ e-mail:ksm@mail.ru
}

\begin{abstract}
This article considers a conversion device that ensures matching of the characteristics of the superconducting inductive (SPIN) energy sources and receivers of electric energy of the traditional performance in a power unit of autonomous perspective of the locomotive. Diesel-electric traction is a wellknown and established technology for railways operators, but this alternative has a considerable non-ecological for the future because only electric traction has a considerable superiority. Besides, diesel-electric engines waste energy when resistive braking is used. This non-regenerative braking decreases the overall efficiency by $\mathbf{1 0 - 2 0 \%}$. With these premises, it is important to develop new strategies to increase the energy efficiency of diesel-electric haulage. To reach a better efficiency, a locomotive with energy storage (battery, super-capacitors) is theoretically proposed. Besides, the possibility of using a lower thermal engine with energy storage devices is considered too. This solution reduces diesel consumption and $\mathrm{CO}_{2}$ emissions while being economically viable. It supposes an efficient energy management because the diesel-electric locomotive could act as a dispersed mobile generation (DMG) unit when working under electric overhead lines, and it can be used as a distributed resource for this specific electric power system.
\end{abstract}

Keywords - energy storage, engine, charge-discharge.

\section{INTRODUCTION}

Increasing energy costs have become a serious concern in our societies and consequently in railway systems. Reducing the energy consumption and improving energy efficiency are important objectives of European Union railway system operators. The opportunities for energy efficiency in railways haulage are a driving strategy (style and coasting), energy storage (in vehicles or offboard), timetables, and energy/speed trade offers. Specifically, diesel-electric trains have serious drawbacks in comparison with electric haulage: diesel locomotives can pack less horsepower, have a greater maintenance cost (due to diesel engine), lower availability (perhaps about 10\%-15\% less than electric locomotives) lifetime, modularity, etc. A diesel railway locomotive is mainly chosen for peripheral areas of transport, where the number of passengers and tonnes of goods are quite limited, and the cost of installing catenary and electric power supplies is too high with respect to traffic needs. The use of diesel is quite limited in EU countries and electric traction should get more and more importance in next decades. Due to these facts diesel locomotives are in excess in some countries and used at a minimum level (maintenance in high-speed lines, secondary lines). In electrified lines, coasting and regenerative braking policies were introduced some decades ago to reduce energy consumption. Regenerative braking is only effective if other trains are available to use the regenerated energy. Successful implementation of regenerative braking requires the use of resistor banks to remove excess energy or inverting substations to regenerate onto the local distribution network, but these possibilities cannot be achieved by diesel-electric haulage.

With the global increase in energy prices, the problem of reducing losses in the transformation, distribution and consumption of energy is becoming a priority for many industries and sectors of the economy, including the transport industry, as one of the most energy-intensive sectors of the economy. In this regard, in the field of transport energy, the task of reducing fuel consumption by autonomous vehicles becomes urgent, with a number of stringent requirements: high profitability, environmental friendliness, reliability, the ability to work on cheap fuels, low maintenance costs, and low weight and size parameters.

Fortunately, recent developments in energy storage devices, particularly supercapacitors and flywheels [I, 2] have made energy storage a viable alternative to apply to railway systems and specifically for diesel-electric units. Energy storage devices can be used to improve energy efficiency by storing regenerated energy from conventional resistive braking. This paper explores the possibilities and use of energy storage in diesel-electric systems, using a real intercity passenger train "DP1A-405" coaches series unit with dieselelectric or electric traction units, used in medium speed tracks.

According to experts [1,2], the possibilities of diesel engines in terms of increasing the specific power, reducing 
fuel costs and reducing harmful emissions into the atmosphere are close to the limit. On the other hand, machine-building enterprises can now offer transport, quite economical and reliable gas turbine engines (GTE) that are capable of operating on cheap fuels can reduce harmful emissions to the atmosphere and substantially reduce the operating costs of transport enterprises. In addition, the use of GTE on locomotives will allow the creation of autonomous locomotives of the same power as electric locomotives, which will significantly reduce the downtime of railcars at the stations connecting electrified and non-electrified railways.

For these reasons, interest in gas turbine locomotives and turbo trains has been reviving in various countries, including Russia [1, 3, 4], long-term research and development programs have been adopted.

The disadvantage of GTE is the significant dependence of the economy and the amount of harmful emissions in the atmosphere from the developed capacity. One of the ways to solve the problem is the use of energy storage devices that will ensure the operation of the GTE with a load close to the nominal, increasing its economy, and the excess energy in the modes of shared traction loads of energy - accumulate [5]. In the future, this energy can be used both for traction needs (for example, to increase the power of the locomotive in excess of the power of the prime mover under particularly severe traffic conditions) and for auxiliary needs (shunting movements and feeding of auxiliary electrical equipment without GTE startup, GTE start-up).

The super-conductor inductive energy storage (SIES) possesses the best indicators among possible prospective versions of high energy capacity storage devices (300 MJ and more), which is characterized by high specific energy intensity, practically unlimited number of charge-discharge cycles and the possibility of unlimitedly long-term energy storage [6], [7].

There are known mathematical methods for modeling the state of objects: graphical (using probabilistic papers and nomograms) and analytical (the maximum likelihood method, the method of moments, quantiles, tabular, Boolean algebras, probabilistic-statistical, modeling by the Markov process) [3, 4]. Modeling by the Markov process is one of the fundamental methods, which has drawbacks - the rigid exponentially of the distribution laws of a random variable and the fact that the elements of the system can only be in two states (workable and inoperative).here are known mathematical methods for modeling the state of objects: graphical (using probabilistic papers and nomograms) and analytical (the maximum likelihood method, the method of moments, quantiles, tabular, Boolean algebras, probabilistic-statistical, modeling by the Markov process) [3, 4]. Modeling by the Markov process is one of the fundamental methods, which has drawbacks - the rigid exponentially of the distribution laws of a random variable and the fact that the elements of the system can only be in two states (workable and inoperative).

In the case of modeling the GTE state of a locomotive, the most probable statistical method is the one that was used as the basis for the model of the technical state of the traction motor of a locomotive [2].

\section{PROBLEM DEFINITION}

In the field of transport energy, the task of reducing fuel consumption by autonomous vehicles becomes urgent, with a number of stringent requirements: high profitability, environmental friendliness, reliability, the ability to work on cheap fuels, low maintenance costs, low weight and weight parameters. The best indicators among possible perspective versions of high-energy storage (300 MJ and more) have a superconducting inductive energy storage (SIES), which is characterized by a high specific energy intensity, an almost unlimited number of charge-discharge cycles and the possibility of unlimitedly long-term energy storage. SIES, as a receiver and source of energy, has the following features: slowly varying current and low voltage. These features are determined by the very low ohmic resistance of the chargedischarge circuit and make it difficult to directly connect to sources and receivers of traditional energy (generators and motors).

Supercapacitors (SC), flywheels and Super magnetics Energy Storage (SMES) are new components that can be used for short-duration energy storage [3, 4]. For example, the University of Texas at Austin Center for Electromechanics (UT-CEM) is currently developing an Advanced Locomotive Propulsion System (ALPS) based on flywheels as part of the Next Generation High-Speed Rail program sponsored by the Federal Railroad Administration (FRA) [5] but flywheels are now an expensive alternative for existing diesel-electric locomotives. Indeed, is it well known that SMES are under development and are very expensive devices? French railways (SNCF) develop other hybrid-locomotive platform based on an old switcher locomotive (class BB63000) with fuel cell, $\mathrm{SC}$, batteries, and flywheels [6].

On the contrary, supercapacitors are a viable alternative from economical and technical viewpoints. The advantages of these components are the stored energy density $(\mathrm{Wh} / \mathrm{kg}$ ) and the volume. The currently available supercapacitors are up to some kilo-Farads (for example, Maxwell Technologies) and their volume is quite reduced.

In comparison to standard batteries, the energy density of supercapacitors is lower by an average factor of 10 . However, their energy density is compatible with a large range of power applications that need high instantaneous power during short periods of time.

The above characteristics of power demand are typically found in transportation systems, and specifically in dieselelectric haulage. Perhaps the main advantage in the use of supercapacitors in comparison with batteries is their lifetime and the number of cycles of storage. Table I presents the main characteristics of supercapacitor and battery energy storage systems.

The objective of this paper is to present how super capacitive and battery storage can be used for increasing the energy efficiency in a diesel-electric railway system and helping in this way in the use of diesel-electric traction in secondary itineraries. Two different solutions will be presented to reach that goal: recovery of braking energy, and 
storage with changes in the diesel engine size and uses.

TABLE I. CHARACTERISTICS OF BATTERYS AND SC

\begin{tabular}{|l|l|l|}
\hline Parameter & Battery & Battery \\
\hline Energy $(\mathrm{Wh} / \mathrm{kg})$ & $10-600$ & $1-10$ \\
\hline Number ofcycles & 1000 & 500000 \\
\hline
\end{tabular}

\section{THEORY}

The SIES charge from the rectifier installed at the output of the SG is most expediently carried out through an intermediate capacitive energy storage (CS) [1]. In this case, the SIES charge will proceed in stages, assuming the nature of a pulsed accumulation of energy. The advantages of these variants consist in the fact that the SG is freed from working with large, slowly varying currents, as a result of which it becomes possible to flexibly regulate the magnitude and duration of the additional load of the gas turbine engine depending on various external conditions. With sufficiently frequent charge pulses of the intermediate CS, the GTE, because of its inertia, will not perceive the impulse, but some average additional load.

In the case of a direct connection of the CS to the output of the rectifier, current surges during the charging process will substantially exceed the values permissible for power semiconductor switches that perform switching. If active resistances are used to limit these shots, this will lead to a significant decrease in the efficiency of the process of charging the intermediate CS, and hence SIES. The circuits using current-limiting reactors are more effective in this respect. Studies conducted earlier showed that the best characteristics are the circuit with the inclusion of currentlimiting reactors after the rectifier device. The process of charging SIES occurs in two stages: the charge of the intermediate CS from the SG through the HC; discharge of intermediate CS on SIES.

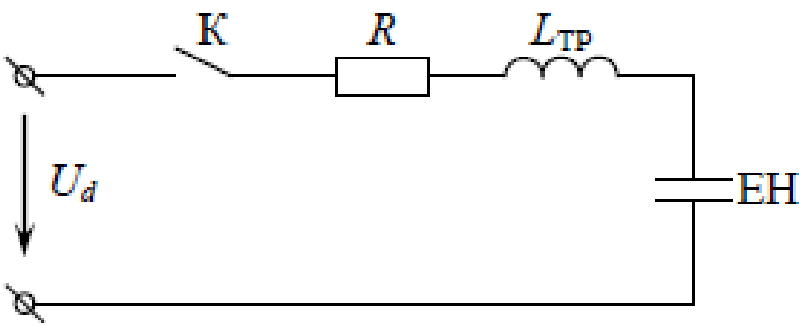

Fig. 1. The replacement circuit of the charging chain of an intermediate CS

In analyzing the operation of the circuit, the following assumptions are made:

Active-inductive resistances included on the side of the alternating current in each phase of the charger, and the active resistances of the wires are assumed to be zero.

The voltage at the output of the rectifier is assumed to be constant.

In the special case, when the reactance of the reactor is close to zero, the expressions for the charge time and the value of the maximum current of the charge CS have the form:

$$
\begin{gathered}
t_{3}=T=\pi \cdot \sqrt{L_{m p}} \cdot C ; \\
I_{m}=U_{d} \cdot \sqrt{\frac{C}{L_{m p}}} .
\end{gathered}
$$

It should be noted that when the current-limiting reactor is turned on after rectifying diodes, the intermediate CS will be charged up to twice the rectifier output voltage. Then the energy accumulated in the intermediate CS in one charge cycle will be:

$$
W=\frac{C \cdot U_{K}^{2}}{2}=2 \cdot C \cdot U_{d}^{2},
$$

where $U_{K}$ - voltage at the intermediate CS at the end of the charge.

From (1) and (2) we determine the time of the charge of the intermediate $\mathrm{CS}$ as a function of the voltage at the output of the rectifier, the maximum value of the current in the process of charge, and the capacity of the storage:

$$
t_{c h}=\pi \cdot \frac{C \cdot U_{d}^{2}}{I_{m}} .
$$

Taking into account the switching time of the semiconductor devices from the nonconductive to the conducting state and back, the minimum charging and discharge time $\mathrm{EH}$ for the efficient operation of the converter will be an order of magnitude larger, let us denote it $t_{\text {swit }}$.

The minimum value for the intermediate CS capacitance will be limited to the minimum allowable charge time equal to $\mathrm{t}_{\text {swit }}$. From (4) we obtain the expression for capacity:

$$
C \geq \frac{t_{\text {swit }}}{\pi} \cdot \frac{I_{m}}{U_{d}} .
$$

From (2), we can determine the inductance of a currentlimiting reactor as a function of the capacity of the intermediate CS:

$$
L_{r c}=\frac{U_{d}^{2}}{I_{m}^{2}} \cdot C
$$

The expression for the charge power of the intermediate EH is obtained by dividing expression (3) on (4):

$$
P_{3}=\frac{W}{t_{3}}=\frac{2}{\pi} \cdot I_{m} \cdot U_{d} .
$$

It is seen from expression (7) that the power at the charge of the intermediate CS depends on the voltage at the output of the rectifier and on the maximum permissible current in the process of charging and does not depend on the capacity of the intermediate $\mathrm{EH}$ and on the inductance of the current-limiting reactoror. 


\section{EXPERIMENTAL RESULTS}

Determination of the parameters of the converter circuit.

Let us consider an example of calculation with the following initial data:

a voltage on an exit of the rectifier $U_{d}=500 \mathrm{~B}$;

$\max$ current SIES $i_{\text {SIES } \max }=2000 \mathrm{~A}$;

maximum permissible current surge with charge of intermediate CS;

$$
I_{m}=2000 \mathrm{~A} ;
$$

minimum charge and discharge time $\mathrm{EH} t_{\mathrm{Swit}}=1 \mathrm{~ms}$;

voltage at intermediate CS with SIES discharge $U_{0}=540$ $V$ (from the supply condition of the AVI, at the output of which the three-phase voltage is the value $220 \mathrm{~V}$ );

range of voltage ripple in the intermediate $\mathrm{CS}$ during discharge SIES $\Delta U=0,05 \cdot U_{0}=27 \mathrm{~V}$.

Determination of the minimum capacitance value of the intermediate CS converter from the expressions obtained above:

when the intermediate CS is charged from the SG through the $\mathrm{HC}$ (по (5)):

$$
C \geq \frac{\text { tswit }}{\pi} \cdot \frac{I_{m}}{U_{d}}=\frac{1 \cdot 10^{-3}}{\pi} \cdot \frac{2000}{500}=1270 \mathrm{mkF} .
$$

When the intermediate CS is discharged to SIES:

$$
C \geq \frac{t_{\text {SIES max }} \cdot t_{\text {swit }}}{2 \cdot U_{d}}=\frac{2000 \cdot 1 \cdot 10^{-3}}{500 \cdot 2}=2000 \mathrm{mkF} .
$$

At a charge of intermediate CS from SIES:

$$
C \geq \frac{t_{\text {SIES max }} \cdot t_{\text {nep }}}{\Delta U}=\frac{2000 \cdot 1 \cdot 10^{-3}}{27}=74000 \mathrm{mkF} .
$$

\section{DISCUSSION OF RESULTS}

In the SPIN charging mode, the intermediate CS should be calculated for a voltage of $2 U_{d}=1000 \mathrm{~V}$, in the discharge mode $-U_{0}=540 \mathrm{~V}$.

The parameters of the elements of the converter circuit essentially depend on the characteristics of the power source and the energy consumers. For the proposed variant of the initial data, in the case of creating a converter with a single intermediate CS, its capacity must be selected from the SIES discharge mode, and the voltage from the charge mode.

According to formula (6), the inductance of the currentlimiting reactor is directly proportional to the capacity of the intermediate CS. If we choose the intermediate CS capacitance by the SIES discharge mode, this will lead to an increase in inductance and, accordingly, the mass-size parameters and cost of the current-limiting reactor in the charge regime almost forty times for the proposed variant of the initial data.

\section{CONCLUSIONS}

Based on the foregoing, we can conclude that two separate schemes are necessary: for SPIN charge and for SPIN discharge, with two different intermediate CS. This will lead to a decrease in the inductance of the current-limiting reactor, since it will be determined by the capacitance of the intermediate CS, obtained from the condition that its discharge time be limited to SIES.

The inductance of the current-limiting reactor will be (6):

$$
L_{r e q}=\frac{U_{d}^{2}}{I_{m}^{2}} \cdot C=\frac{500^{2}}{2000^{2}} \cdot 2000 \cdot 10^{-6}=0.0125 b=m H
$$

When feeding onboard consumers, the required type of current-voltage characteristic at the input is possible only if there is an intermediate converter of the slowly varying current of the SIES into a voltage maintained practically constant. Such converter can be a CS of low energy intensity.

The parameters of the elements of the converter circuit essentially depend on the characteristics of the power source and the energy consumers. An option is possible in which it would be more expedient to create two independent converters - separately for the charge and discharge mode with two intermediate CS.

\section{References}

[1] V.I.Sopov, V.V. Biryukov, Y.A. Prokushev, Y.A. Rylov, "Analysis of power supply systems for rolling stock with various schemes of traction networks", Abstract journal of VINITI RAS: Transport, Science, Engineering, Management, vol. 2 (2008), pp. 49-53, 2008.

[2] E.N. Bykov, B.V.Panin, V.N.Pupynin, Traction networks of subways, Moskow: Transport, 1987.

[3] O.I. Sablin, "Improving the efficiency of energy recovery in the electric transport system with limited traction power supply", Technological audit and production reserves, vol. 5/1, pp. 21-26, 2014.

[4] V. G. Sysenko VG, D.A. Bosvit, E.N. Kosarev, "Improvement of the methodology for calculating the distributed traction power-supply system with an amplifying point", Power electronics and power engineering, vol. 9, pp. 8-18, 2014.

[5] V.V. Biryukov, A.V. Kulekina, "The calculation features of the electrical energy storage devices parameters in transport", IFOST 2016, pp. 41-43, June 2016 [The 11 International forum on strategic technology, p. 596, 2016].

[6] V.I. Sopov, N.I. Schurov, Y.A. Prokushev and A.A. Shtang, "Increasing the efficiency of the use of electrical energy in the sub-subsystem of electric transport", Improvement of technical means of electric transport, vol. 3 (29), pp. 115-123, Jule 2002 [Digest of scientific works of the NSTU, p. 189, 2002].

[7] M.V. Tarnizhevsky, D.K. Tomlyakovich, Design of power supply devices for trams and trolleybuses, Moskow: Transport, 1986.

[8] K.G. Marquardt, Electrosupply of Electrified Railways, Moskow: Transport, 1982.

[9] M.A. Sleptsov, Fundamentals of Electric Transport, Moskow: Academy, 2006.

[10] L.A. Bessonov, Theoretical bases of electrical engineering, Moscow: Higher School, 1973. 